\title{
Epidemiological Characteristics and Transmission Patterns of COVID-19 Cases Among Children and Adolescents Aged 0-18 Years in South Korea
}

\author{
Jinhwa Jang', Myung-Jae Hwang', Yoo-Yeon Kim', Shin Young Park', Myeongsu Yoo', Seong-Sun Kim ', \\ Sangwon Lee $\mathbb{D}^{2}$, Donghyok Kwon (D) \\ 'Division of Public Health Emergency Response Research, Korea Disease Control and Prevention Agency, Cheongju-si, Chungcheongbuk-do, Republic \\ of Korea; ${ }^{2}$ Director General for Public Health Emergency Preparedness, Korea Disease Control and Prevention Agency, Cheongju-si, \\ Chungcheongbuk-do, Republic of Korea \\ Correspondence: Donghyok Kwon, Division of Public Health Emergency Response Research, Korea Disease Control and Prevention Agency, \\ 187, OsongSaengmyeong 2-ro, Osong-eup, Heungdeok-gu, Cheongju-si, Chungcheongbuk-do, 28I59, Republic of Korea, Tel +82-43-7|9-7730, \\ Fax +82-43-719-7749, Email vethyok@korea.kr
}

Background: In South Korea, the epidemiological characteristics of children and adolescents infected with severe acute respiratory syndrome coronavirus-2 (SARS-CoV-2) have not been reported to date. The purpose of this study was to identify major epidemiological characteristics and transmission patterns of coronavirus disease 2019 (COVID-19) in children and adolescents.

Methods: This study was conducted through a system integrated in an epidemiological investigation by the Korea Disease Control and Prevention Agency from January 20, 2020, to June 5, 2021. We analyzed the epidemiological characteristics of 14,967 children and adolescents with COVID-19 according to the age groups and transmission age patterns of 3721 infector-infectee pairs in South Korea.

Results: Among the total confirmed COVID-19 cases, 14,967 patients were aged 0-18 years. The most affected age group among children and adolescents were those aged 16-18 years (3589, 24.0\%). For all age groups, the infection route through friends and family members (31.9\%) was the highest. For the contact age pattern analysis, infection from infectors aged 30-49 years to infectees aged 012 years showed a statistically significant relation $(p<0.001)$ compared to that in other age groups. On the other hand, among the infectees aged 13-15 years and 16-18 years were significantly related with adolescents aged 10-19 years $(p<0.05)$.

Conclusion: These results suggest that adolescents aged 13-18 years were more infected with COVID-19 than those aged 0-12 years. Furthermore, they are particularly more likely to be infected by friends and family members. Besides, in patients aged 13-18 years, transmission of SARS-CoV-2 was more common from adolescents to adolescents than from adults to adolescents. This research will provide scientific evidence for school policies and vaccine strategies for COVID-19 prevention in children and adolescents.

Keywords: coronavirus disease-2019, severe acute respiratory syndrome coronavirus-2, incidence rate, mortality

\section{Introduction}

Coronavirus disease-2019 (COVID-19), originated in Wuhan, China, in December 2019 and was declared as a Public Health Emergency of International Concern (PHEIC) by the World Health Organization in January 2020. As of June 16, 2021, more than 177 million cases have been confirmed, with more than 3.83 million deaths attributed to COVID-19 in the world. ${ }^{1}$ One of the most concerning issues is the extent to which COVID-19 affects children and adolescents and the role of major transmission routes. Children and adolescents below 18 years of age constituted approximately $8 \%$ of all COVID-19 cases reported by countries in 2020, despite representing $29 \%$ of the global population. ${ }^{2}$ In the United States, 4,064,365 children with COVID-19 were reported, which represented $14.2 \%$ of the total 28,645,258 cases until July 8 , 2021. The overall incidence rate in the US is 5400 cases per 100,000 children. Children accounted for $1.3 \%$ to $3.6 \%$ of the total reported hospitalizations, and $0.1 \%$ to $1.9 \%$ of all COVID-19 confirmed children were hospitalized. Concerning 
mortality in 43 states of the US, children accounted for $0 \%$ to $0.25 \%$ of all COVID-19 deaths, and eight states reported zero child deaths. ${ }^{3}$ Other studies have found that the overall risk of death or severe disease from COVID-19 is very low in children. ${ }^{4}$ A comprehensive analysis of hospital admissions and reported deaths across England suggested that COVID-19 carries a lower risk of death or need for intensive care among children and adolescents. ${ }^{5}$

In South Korea, COVID-19 outbreak started with imported cases from Wuhan, China, on January 20, 2020 and is still prevalent in the community as of June 2021. By June 5, 2021, there were 143,594 laboratory-confirmed cases and 1971 deaths from COVID-19 in South Korea. ${ }^{6}$ Among these patients, a total of 14,967 laboratory-confirmed cases of COVID19 among children and adolescents aged 0-18 years were reported. In response to the pandemic situation, nonpharmacological interventions, such as quarantine, social distancing, contact tracing, and diagnostic tests were applied, and in February 2021, vaccines were introduced and administered to the elderly (over 65 years old) and vulnerable populations on priority basis. ${ }^{7,8}$ However, since the recent resumption of schools nationwide, the average age of individuals suffering from COVID-19 has been decreasing, and the incidence rate among adolescents is increasing. There are also arguments for an extension of the recommended age range for vaccination, along with universal mitigation strategies, to reduce community transmission, which will help schools operate safely through face-to-face learning. ${ }^{9,10}$

The aim of this study was to evaluate the epidemiological characteristics in terms of demographic factors and transmission patterns among children and adolescents with SARS-CoV-2 to provide scientific evidence for these opinions. Based on the national database, it can be hypothesized that the transmission pattern between the infector and the infectee follows age groups. Through this study we aimed to provide evidence for expanding the recommended age range for vaccination in South Korea.

\section{Materials and Methods}

\section{Study Subjects}

Based on the Infectious Disease Prevention and Control Act, all suspected COVID-19 cases require laboratory confirmation and epidemiologic investigation. This study was based on all laboratory-confirmed cases reported through the integrated system in the epidemiological investigation conducted by the Korea Disease Control and Prevention Agency (KDCA) and local government public health departments. We collected epidemiological and demographic data on 14,967 laboratory-confirmed COVID-19 cases identified in South Korea from January 20, 2020 to June 5, 2021. Some of the data are available for research purposes through the National Infectious Disease Surveillance System website (https://covid19.kdca.go.kr/) according to user permission. Confirmed COVID-19 cases, defined as positive for SARSCoV-2 nucleic acid by using real-time reverse transcriptase-polymerase chain reaction (RT-PCR) assay from nasal and pharyngeal swab specimens or blood samples. This observational study was approved by the institutional review board of Korea Disease Control and Prevention Agency (KDCA, KDCA 2021-04-07-PE-A), and the requirement for informed consent was waived.

\section{Study Design}

The data of confirmed cases consisted of sex, age, reported date, address of reporting agency, residential address, infection route, related clusters, hospitalization, and epidemiological investigation-based information on contacted confirmed cases. The infection routes of confirmed cases were identified based on an in-depth epidemiological survey along with a basic epidemiological survey, and sub-clusters were defined based on the surveyed information. Individual demographic factors, such as sex, age, and residential address were determined based on the epidemiological survey. We gathered personal epidemiological records including severity of illness, days from symptom onset to diagnosis, initial symptom awareness, major infection route and prior contact cases. Children were defined as being under 18 years old and were divided into five categories as follows: infants and toddlers (aged 0-2 years), preschooler (aged 3-6 years), elementary student (aged 7-12 years), middle school student (aged 13-15 years), and high school student (aged 16-18 years) groups. ${ }^{11}$ We calculated the weekly incidence rate per 100,000 people during the COVID-19 pandemic. The total population by age group was obtained from the census survey data collected by Ministry of the Interior and Safety from South Korea in January 2020. Considering the period of offline (face-to-face) and online schooling, which was 
extensively implemented following the social distancing stage in South Korea, we observed weekly patterns of the number of confirmed cases, hospitalization, and school-related cases. We conducted the age comparison among and between 3721 COVID-19 transmission pairs. The case with the earlier report was defined as an infector (prior contact cases), and that with the later report was defined as an infectee (secondary case). ${ }^{12}$ Information on the infectors was tracked by classifying them according to the sub-cluster of confirmed cases in children, and the relationship between the age group of the infectee and the infector was analyzed using splines function. ${ }^{13}$ The size of each point indicates the relative number of pairs in each combination.

\section{Data Analysis}

Associations between the infectee and infector age groups using a generalized linear model (GLM) were analyzed. The level of statistical significance was set at $p=0.05$ and $95 \%$ confidence intervals (CIs) were estimated for the point estimate. R software (version 4.0.2; R Foundation) was used for the statistical analysis.

\section{Results}

\section{Epidemiological Characteristics of COVID-19 Cases Aged 0-18 Years}

From January 20, 2020 to June 5, 2021, a total of 14,967 laboratory-confirmed cases of COVID-19 among children and adolescents aged 0-18 years were reported in South Korea. Of these, the majority (42.0\%) occurred among adolescents aged 13-18 years. High school students (16-18 years) accounted for $24.0 \%$ of the cases; middle school students (13-15 years), 18.0\%; elementary school students (7-12 years), 30.5\%; preschoolers, (3-6 years) 16.7\%; and infants and toddlers ( $0-2$ years), $10.8 \%$ (Table 1 ). Overall, $54.6 \%$ of the cases were male. More than half of the patients were from the metropolitan areas $(9611 ; 64.2 \%)$. Among patients aged 16-18 years, weekly incidence was higher in each successively increasing age group. There was only one severe or critical case in adolescents, and no deaths were reported in children and adolescents aged 0-18 years. Among the children and adolescents aged 0-18 years with available data for these outcomes, 4982 (33.3\%) were hospitalized and 7638 (48.3\%) had symptoms at the time of confirmation. Among the patients aged 0-18 years, the largest percentage of hospitalizations (49.1\%) occurred in children aged 0-2 years. The period from diagnosis to quarantine among all age groups aged 0-18 years was 12 days, with an interquartile range of 11 to 15 days.

The results show the trend of weekly incidence rates according to age groups (Figure 1). Among adolescents aged 1618 years, the weekly incidence (per 100,000 persons) was highest during the week of April 18, 2021 (weekly incidence 10.4). For adolescents aged 13-15 years, weekly incidence was highest during the week of January 24, 2021 (weekly incidence 10.9). Furthermore, in children aged 7-12 years, the weekly incidence was highest during the week of December 13, 2020 (weekly incidence 8.5). Those aged 0-6 years had the highest weekly incidence during the week of December 20, 2020 (weekly incidence 7.0). Time trends in weekly reported incidence among adolescents aged 16-18 years were consistently higher than those in the other age groups during almost all weeks. The incidence among the adolescents aged 13-15 years was higher than that of those aged 16-18 years in the week of August 17, 2020 and January 24, 2021. The trend in incidence among the adolescents aged 16-18 years had distinct and more prominent peaks during the weeks at the end of January and April.

The weekly number of hospitalized patients tended to increase with the number of confirmed patients aged 0-18 years. There was no significant effect on the increase or decrease in the number of confirmed cases, depending on the school's online and offline class periods. Since the beginning of the third wave (November 13, 2020), the number of cases has repeatedly increased and decreased in all age groups (Figure 2). The results show major transmission routes of infection by age group in children (Table 2). The route of infection that showed the highest proportion among all age groups was family-friend gatherings (31.9\%). The family and friend gatherings related route were the highest route of infection in all age groups. The second route of infection was contact with previously confirmed cases (15.6\%) except family and friends. In case of individuals aged 16-18 years, family and friend gatherings (23.3\%) were the major route of infection, however it was lower compared to that in other age groups. The proportion of educational facility clusters $(11.5 \%)$ was higher than that of other age groups among adolescents aged 16-18 years. 
Table I Characteristics of COVID-19 Cases Among Individuals Aged 0-18 Years in South Korea

\begin{tabular}{|c|c|c|c|c|c|c|c|}
\hline \multirow{2}{*}{$\begin{array}{l}\text { Variables } \\
\text { Characteristic }\end{array}$} & \multicolumn{7}{|c|}{ Age Group (Aged 0-18 Years) } \\
\hline & Total & 0-2 Yrs & 3-6 Yrs & $7-12$ Yrs & $13-15$ Yrs & $16-18$ Yrs & p-value* \\
\hline \multicolumn{8}{|c|}{ Total number of patients, $n$ (\%) } \\
\hline & 14,967 & $1623(10.8)$ & $2503(16.7)$ & $4555(30.5)$ & $2697(\mid 8.0)$ & $3589(24.0)$ & $<0.0001$ \\
\hline \multicolumn{8}{|l|}{ Sex, n (\%) } \\
\hline Male & $8179(54.6)$ & $876(54.0)$ & $1326(53.0)$ & $2417(53.1)$ & $1456(54.0)$ & $2104(58.6)$ & $<0.0001$ \\
\hline Female & $6788(45.4)$ & $747(46.0)$ & $1177(47.0)$ & $2138(46.9)$ & $|24|(46.0)$ & |485 (4I.4) & \\
\hline \multicolumn{8}{|l|}{ Province, $\mathbf{n}(\%)$} \\
\hline Metropolitan area & $9611(64.2)$ & $1135(69.9)$ & $1668(66.6)$ & $2896(63.6)$ & $1680(62.3)$ & $2232(62.2)$ & $<0.0001$ \\
\hline Non-metropolitan area & $5356(35.8)$ & $488(30.1)$ & $835(33.4)$ & $1659(36.4)$ & $1017(37.7)$ & $1357(37.8)$ & $<0.0001$ \\
\hline \multicolumn{8}{|l|}{ Incidence rate ${ }^{\dagger}$} \\
\hline & 177 & 166 & 145 & 160 & 199 & 234 & $<0.0001$ \\
\hline \multicolumn{8}{|c|}{ Total number of severe/critical medical condition $\S$} \\
\hline & I & 0 & 0 & 0 & 0 & I & \\
\hline \multicolumn{8}{|l|}{ Total number of deaths } \\
\hline & 0 & 0 & 0 & 0 & 0 & 0 & \\
\hline \multicolumn{8}{|c|}{ Total number of hospitalization, $n$ (\%) } \\
\hline & $4982(33.3)$ & $797(49.1)$ & $890(35.6)$ & $1495(32.8)$ & $763(28.3)$ & $1037(28.9)$ & $<0.0001$ \\
\hline \multicolumn{8}{|c|}{ Initial symptom awareness, $\mathbf{n}(\%)$} \\
\hline Symptomatic & $7638(48.3)$ & $821(50.6)$ & 981 (39.2) & $1950(42.8)$ & $1563(58.0)$ & $2323(64.7)$ & $<0.0001$ \\
\hline Asymptomatic & $6555(46.6)$ & $727(44.8)$ & $1396(55.8)$ & $2397(52.6)$ & $1001(37.1)$ & $1034(28.8)$ & \\
\hline \multicolumn{8}{|c|}{ Days from diagnosis to quarantine, day } \\
\hline Median & 12 & 12 & 12 & 12 & 12 & 12 & $<0.0001$ \\
\hline Interquartile range & $11-15$ & $11-15$ & $11-15$ & $11-15$ & $11-14$ & $11-15$ & \\
\hline
\end{tabular}

Notes: ${ }^{\dagger}$ Number of patients with laboratory-confirmed COVID-19 per 100,000 persons using 2020 Korea Census population estimates. ${ }^{\S}$ COVID-19 patients in isolation being treated with high-flow oxygen therapy, mechanica ventilation, extracorporeal membrane oxygenation (ECMO), and continuous renal replacement therapy (CRRT). ${ }^{*}$-values obtained by comparing the groups using the chi-square test or Fisher's exact test. 


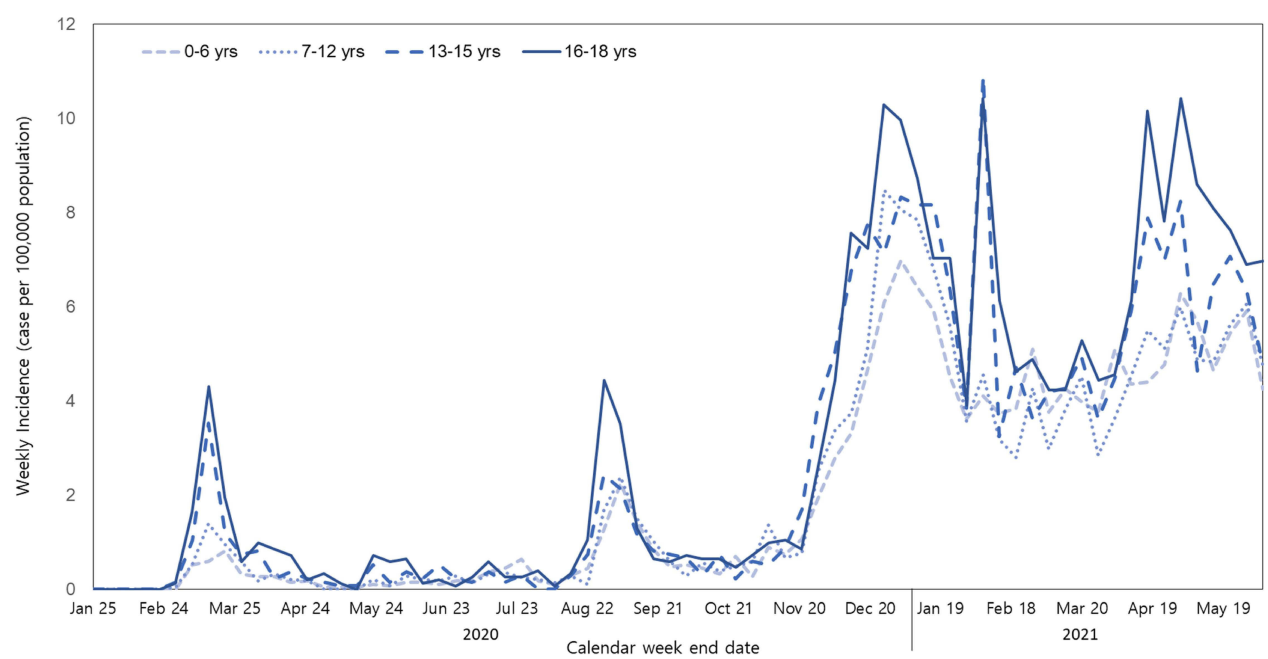

Figure I COVID-19 weekly incidence cases among individuals aged 0-18 years by age group in South Korea, January 20-June 5, 202I.

\section{Contact Age Patterns Among the Infectees Aged 0-18 Years by Age Group}

Among the 3721 confirmed COVID-19 cases, we observed the infectors of all ages and infectees aged 0-18 years for contact patterns. The contact patterns among individuals aged 0-18 years according to the four age groups are shown in Figure 3. A positive trend was observed between the same age groups and the parent age groups. The gray bands present the 95\% confidence intervals around the regression line. Infection from parents aged 30-49 years to children and adolescents aged 0-18 years showed strong interaction compared to that observed in other age groups. In particular, adolescents aged 13-15 years and 16-18 years showed a statistically significant relation between the infectors and the infectees of the same age group than that observed in children below 12 years of age. The infectees aged $0-6$ years were significantly increasing in infectee aged $30-44$ years $(\beta=0.78, p<0.001)$. Among the infectees aged $7-12$ years were significantly increasing in infectee aged 35-49 years $(\beta=0.51, p<0.001)$. Among middle school students aged $12-15$ years were significantly increasing in infectee aged $10-19$ years $(\beta=0.65, p<0.001)$. Also, the high school student aged $16-18$ years were significantly increasing in infectee aged $10-19$ years $(\beta=0.64, p<0.05)$.

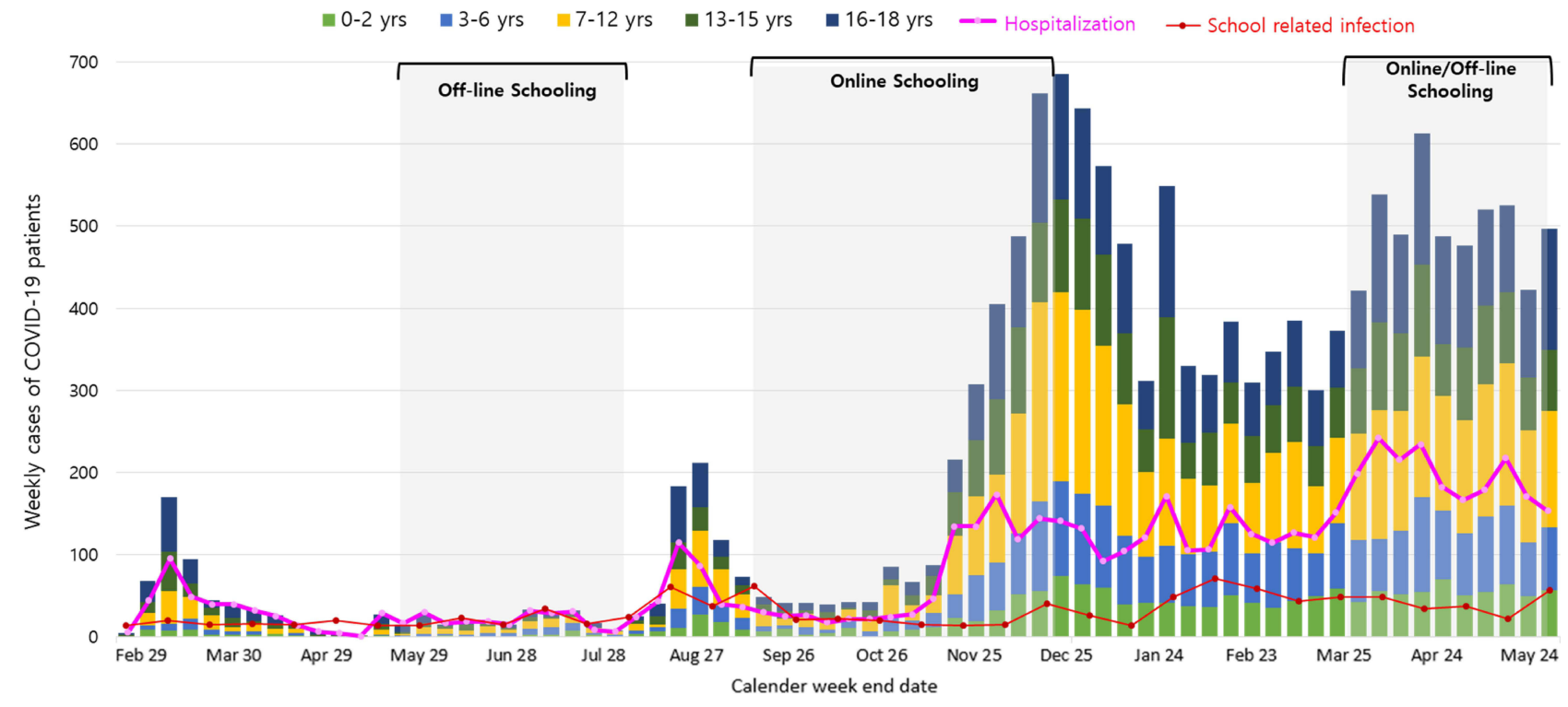

Figure 2 COVID-19 weekly cases among individuals aged 0-18 years by age group in South Korea, January 20-June 5, 202I. 
Table 2 Major Transmission Routes of COVID-19 Cases Among Individuals Aged 0-18 Years by Age Group

\begin{tabular}{|c|c|c|c|c|c|c|c|}
\hline \multirow{2}{*}{$\begin{array}{l}\text { Variables } \\
\text { Major Infection Routes }\end{array}$} & \multicolumn{7}{|c|}{ Age Group (Age 0-18 Years), n (\%) } \\
\hline & Total & 0-2 Yrs & 3-6 Yrs & $7-12$ Yrs & 13-15 Yrs & $16-18$ Yrs & $p$-value* \\
\hline Family Friends gatherings clusters & $4774(31.9)$ & $650(40.0)$ & $930(37.2)$ & $1604(35.2)$ & $755(28.0)$ & $835(23.3)$ & $<0.05$ \\
\hline Contact with confirmed cases & $2330(15.6)$ & $254(15.7)$ & $426(17.0)$ & $736(16.2)$ & $391(14.5)$ & $523(14.6)$ & $<0.05$ \\
\hline Religious facility clusters & $1650(11.0)$ & $121(7.5)$ & $202(8.1)$ & $5 I I(I I .2)$ & $405(15.0)$ & $4 I I(I I .5)$ & $<0.05$ \\
\hline Educational facility clusters & $1639(11.0)$ & $158(9.7)$ & $266(10.6)$ & $377(8.3)$ & $285(10.6)$ & $553(15.4)$ & $<0.05$ \\
\hline Overseas imported cases & $1082(7.2)$ & $107(6.6)$ & $184(7.4)$ & $331(7.3)$ & $184(6.8)$ & $276(7.7)$ & $<0.05$ \\
\hline Other public facility clusters & $757(5.1)$ & $100(6.2)$ & $119(4.8)$ & $210(4.6)$ & $112(4.2)$ & $216(6.0)$ & $<0.05$ \\
\hline Workplace clusters & $865(5.8)$ & $98(6.0)$ & $163(6.5)$ & $320(7.0)$ & $150(5.6)$ & $134(3.7)$ & $<0.05$ \\
\hline Nursing facility.Hospital clusters & $297(2.0)$ & $35(2.2)$ & $64(2.6)$ & $101(2.2)$ & $43(1.6)$ & $54(1.5)$ & $<0.05$ \\
\hline Correction·Military facility clusters & $37(0.2)$ & $3(0.2)$ & $4(0.2)$ & $4(0.1)$ & $13(0.5)$ & $13(0.4)$ & $<0.05$ \\
\hline Under investigation & $1537(10.2)$ & $97(5.9)$ & $145(5.6)$ & $362(7.9)$ & $359(13.2)$ & $574(15.9)$ & $<0.05$ \\
\hline
\end{tabular}

Note: ${ }^{*} p$-values obtained by comparing the groups using the chi-square test or Fisher's exact test.

\section{Discussion}

To the best of our knowledge, this is the first study on the epidemiological and demographic characteristics and transmission dynamics of COVID-19 in children and adolescents from South Korea. We analyzed the epidemiological characteristics of 14,967 laboratory-confirmed COVID-19 cases identified in South Korea from January 20, 2020 to June 5, 2021. Most of those aged 0-18 years were likely to expose themselves to family members or friends with COVID-19. Supportive evidence of such transmission pathways has been reported by previous studies. ${ }^{12}$ The weekly incidence of COVID-19 and the percentage of patients among children and adolescents increased during the review
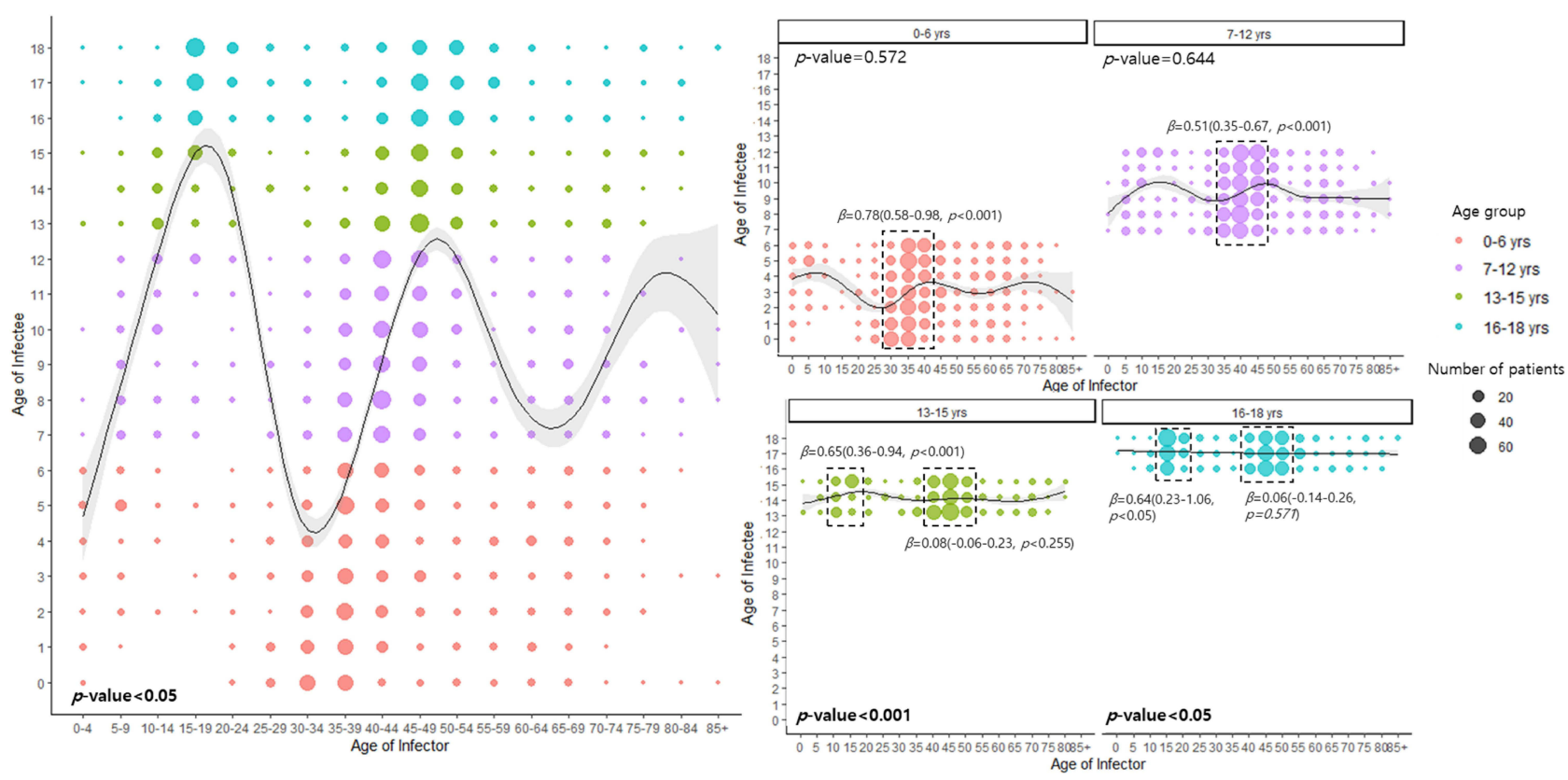

Figure 3 Contact age patterns among infector-infectee pairs aged 0-18 years, according to age groups. The $95 \%$ confidence intervals of the fitted value are shown in grey $(\beta$ : point estimate ( $95 \%$ confidence interval, $p$-value), statistically significant values ( $p$-value) bold for each age groups). 
period, with an outbreak peak in January and April 2021. In particular, the cumulative incidence was highest in adolescents aged 16-18 years compared to that observed in other age groups. The higher cumulative incidence rate of COVID-19 in adolescents is the reason why school closures are considered a key intervention for controlling COVID-19. COVID-19 mortality among patients aged 0-18 years was zero because no deaths were reported. However, other countries, including the USA, UK, Italy, Germany, Spain, and France, have reported COVID-19 deaths in children. ${ }^{14}$ The reason for patients aged 0-2 years having the highest hospitalization rate is that all COVID-19 patients shall receive inpatient treatment at an infectious disease control institution, a hospital specialized in infectious diseases, except patients who self-treat at home, according to the South Korea Infectious Disease Control and Prevention Act. The patients aged 0-2 years, the COVID-19 high risk group, were excluded from self-treatment and were mostly hospitalized. For this reason, while the hospitalization rate in infants and young children was high, there were no severe or fatal cases in this age group. These patterns are similar in other studies, despite the higher incidence of COVID-19 in adolescents, infants seem to be the most vulnerable due to a high hospitalization rate. ${ }^{15}$

As a result of contact age patterns, preschoolers (0-6 years) and elementary school students (7-12 years) were significantly related with their parent age groups (30-49 years). On the other hand, middle school students (13-15 years) and high school students (16-18 years) were significantly related with their friends age groups (10-19 years). This pattern of transmission in adolescents reflected a specific education system in South Korea. Students in the adolescent age group in South Korea are the most active in appearing for entrance exams and are engaged in private as well as public education. Because of this specific education system, it is estimated that the infection spread in the same age group through education-related facilities, such as private academies and classrooms. A study of COVID-19 transmission in England showed a significant correlation between outbreaks in educational settings and COVID-19 incidence in the community. ${ }^{16}$ In China, children with COVID-19 were mainly infected via family clustering and had a long incubation period. For example, in another study from China, $66 \%$ of pediatric patients were infected via family members with COVID-19. ${ }^{17}$ Additionally, children tend to have more social contact in schools than adults, therefore contributing more to COVID-19 transmission than adults between the same age group. ${ }^{18}$ Unlike other countries, in Korea, the source of infection is confirmed through active preemptive testing of those in close contact. In addition, quarantine policies have been implemented to minimize the risk of infection caused by the index case. However, despite these nationwide efforts, if the spread of COVID-19 in schools, where educational activities occur, worsens, the overload causes aggravation of domestic medical resources, and the admission and aggravation rates are expected to increase. Therefore, in this pandemic situation, it is necessary to grasp the pattern of the domestic epidemic from a macroscopic point of view and prepare countermeasures through active implementation of quarantine rules in the local community through cooperation of related ministries.

In South Korea, the Epidemic Intelligence Support System (EISS) has been developed to track the route of COVID-19 infections and continuously revise relevant information. In this study, it was confirmed that the pattern of transmission by contact with a confirmed case clearly changes with the pattern of COVID-19 epidemic in South Korea. However, there were some limitations. First, there was still uncertainty about the route of infection and information on confirmed cases obtained from epidemiological investigations. Therefore, to overcome this uncertainty, we conducted our study based on the latest information from the National Infectious Disease Surveillance System. Second, we analyzed the relationship between the infector-infectee pair by defining a prior confirmed case based on the onset date and information on the infection route when the infection spread in a specific group. To overcome the information bias, only accurate information was obtained by examining the causal relationship between outbreaks when a large number of previously confirmed cases were enrolled. Third, the admission rate was calculated using only hospitalization information, without considering the severity of symptoms. In fact, if information on the severity of confirmed cases was available, the correlation of severity according to the level of close contact might have been evaluated using the relationship of prior confirmed cases.

Nevertheless, this study had several strengths. First, the scientific rationale for selecting a priority target for establishing a COVID-19 vaccination strategy has been suggested. In South Korea, vaccine administration has been prioritized for medical staff and susceptible populations; currently, the authorities are planning to vaccinate persons aged 18-49 years. However, with the recent changes in COVID-19 transmission and mode of spread, school-age vaccination must be initiated quickly. Furthermore, the age range for vaccination must be expanded by securing more vaccine volumes. Second, our study 
suggested that more preemptive tests should be conducted for adolescent age groups with high activity and a wide range of behaviors, and quarantine guidelines should be strengthened in education-related facilities where students frequently visit. In addition, in case of confirmed cases among school-going children, the majority of infections occur through transmission between family members and acquaintances; therefore, when the infection spreads, it spreads faster and has a wider range of contacts than that in other age groups. Therefore, it is necessary to isolate confirmed patients before the onset of symptoms through preemptive testing to block the spread of COVID-19. Finally, this study suggested that it is necessary to identify the characteristics of major infected groups during the COVID-19 pandemic period and redefine the community risk index regarding the transmission pattern. In particular, this study suggests that the level of social distancing should be established according to the number of confirmed cases across the country as well as the main transmission populations. This would be a huge contributor to reducing the risk of COVID-19 transmission.

\section{Conclusion}

Our results show the epidemiological characteristics and contact patterns of COVID-19 cases aged 0-18 years in South Korea. The total number of patients aged 0-18 years was $10.4 \%$ among all confirmed cases in South Korea. Among these cases, the majority (42.0\%) occurred among adolescents aged 13-18 years. In particular, the patients aged 13-15 years and 16-18 years showed a statistically significant relation between the infector and the infectee in the same age group. In addition, the proportion of educational facility clusters in adolescents aged 16-18 years is higher than that of other adolescent age groups. The relatively high infectivity among adolescents in educational facilities should be considered carefully when making decisions around school re-openings, as infected children can pass the virus to their friends and family members. Further studies are needed to assess the efficacy of COVID-19 vaccination in adolescents aged 12-18 years in South Korea.

\section{Data Sharing Statement}

The dataset used in this study was collected by the Korea Disease Control and Prevention Agency and part of them (demographics, major symptoms, etc.) are available for research purpose through the National Infectious Disease Surveillance System website (https://covid19.kdca.go.kr/).

\section{Ethics Approval}

This study was approved by the institutional review board of Korea Disease Control and Prevention Agency (KDCA 2021-04-07-PE-A), and informed consent requirement was waived.

\section{Acknowledgments}

We thank all the healthcare workers involved in the diagnosis and treatment of patients with COVID-19 in South Korea.

\section{Author Contributions}

JJ and DK designed the study and took responsibility for the integrity of the data and accuracy of the data analysis. JJ and MJH performed statistical analyses and drafted the manuscript and figures. YYK, SYP, MY, SSK, and SL provided reviews and comments for the study. All authors made a significant contribution to the work reported, whether that is in the conception, study design, execution, acquisition of data, analysis and interpretation, or in all these areas; took part in drafting, revising or critically reviewing the article; gave final approval of the version to be published; have agreed on the journal to which the article has been submitted; and agree to be accountable for all aspects of the work.

\section{Disclosure}

The authors report no conflicts of interest in this work.

\section{References}

1. COVID-19 weekly epidemiological update; 2021. Available from: https://www.who.int/publications/m/item/weekly-epidemiological-update-oncovid-19. Accessed June 29, 2021. 
2. COVID-19 weekly epidemiological update; 2021. Available from: https:/www.who.int/publications/m/item/weekly-epidemiological-update. Accessed June 29, 2021.

3. American Academy of Pediatrics. Children and COVID 19: state data report. Available from: https:/services.aap.org/en/pages/2019-novelcoronavirus-covid-19-infections/children-and-covid-19-state-level-data-report/. Accessed July 29, 2021.

4. Ludvigsson JF. Systematic review of COVID-19 in children shows milder cases and a better prognosis than adults. Acta Paediatrica. 2020;109 (6):1088-1095. doi:10.1111/apa.15270

5. Smith C, Odd D, Harwood R, et al. Deaths in children and young people in England after SARS-CoV-2 infection during the first pandemic year. Nat Med. 2021;1-8. doi:10.1038/s41591-021-01578-1

6. Jang J, Kim Y, Kim YY, et al. Coronavirus Disease-19 (COVID-19) one-year outbreak major cluster infection report as of January 19, 2021, in the Republic Of Korea. Public Health Wkly Rep. 2020;14(9):482-495.

7. Park Y, Huh IS, Lee J, et al. Application of testing-tracing-treatment strategy in response to the COVID-19 outbreak in Seoul, Korea. J Korean Med Sci. 2020;35(45):e396. doi:10.3346/jkms.2020.35.e396

8. Jeon M, Kim J, Oh CE, et al. Adverse events following immunization associated with coronavirus disease 2019 vaccination reported in the mobile vaccine adverse events reporting system. J Korean Med Sci. 2021;36(17):e114. doi:10.3346/jkms.2021.36.e114

9. Leidman E, Duca LM, Omura JD, et al. COVID-19 trends among persons aged 0-24 years-United States, march 1-December 12, 2020. Morb Mortal Wkly Rep. 2021;70(3):88-94. doi:10.15585/mmwr.mm7003e1

10. Havers FP, Whitaker M, Self JL, et al. Hospitalization of adolescents aged 12-17 years with laboratory-confirmed COVID-19-COVID-NET, 14 states, March 1, 2020-April 24, 2021. Morb Mortal Wkly Rep. 2021;70:851-857. doi:10.15585/mmwr.mm7023e1

11. Kim EY, Ryu B, Kim EK, et al. Children with COVID-19 after reopening of schools, South Korea. Pediatr Infect Vaccine. 2020;27(3):180-183. doi:10.14776/piv.2020.27.e23

12. Yi S, Choe YJ, Ahm S, et al. Geospatial analysis of age-specific SARS-CoV-19 transmission patterns in households, Korea. J Korean Med Sci. 2021;36(8):e63. doi:10.3346/jkms.2021.36.e63

13. Adam DC, Wu P, Wong JY, et al. Clustering and superspreading potential of SARS-CoV-2 infections in Hong Kong. Nat Med. $2020 ; 26: 1714-1719$. doi:10.1038/s41591-020-1092-0

14. Bhopal SS, Bagaria J, Olabi B, et al. Children and young people remain at low risk of COVID-19 mortality. Lancet Child Adolesc Health. 2021;5 (5):E12-E13. doi:10.1016/S2352-4642(21)00066-3

15. Rankin DA, Talj R, Howard LM, et al. Epidemiologic trends and characteristics of SARS-CoV-2 infections among children in the United States. Curr Opin Pediatr. 2021;33:114-121. doi:10.1097/MOP.0000000000000971

16. Ismail SA, Saliba V, Lopez BJ, et al. SARS-CoV-2 infection and transmission in educational settings: a prospective, cross-sectional analysis of infection clusters and outbreaks in England. Lancet Infect Dis. 2021;21:344-353. doi:10.1016/S1473-3099(20)30882-3

17. Guo CX, He K, Yin JY, et al. Epidemiological and clinical features of pediatric COVID-19. BMC Med. 2020;18(1):250. doi:10.1186/s12916-020$01719-2$

18. Davies NG, Klepac P, Yang L, et al. Age-dependent effects in the transmission and control of COVID-19 epidemics. Nat Med. $2020 ; 26: 1205-1211$. doi:10.1038/s41591-020-0962-9

Risk Management and Healthcare Policy

Dovepress

\section{Publish your work in this journal}

Risk Management and Healthcare Policy is an international, peer-reviewed, open access journal focusing on all aspects of public health, policy, and preventative measures to promote good health and improve morbidity and mortality in the population. The journal welcomes submitted papers covering original research, basic science, clinical \& epidemiological studies, reviews and evaluations, guidelines, expert opinion and commentary, case reports and extended reports. The manuscript management system is completely online and includes a very quick and fair peer-review system, which is all easy to use. Visit http://www.dovepress.com/testimonials.php to read real quotes from published authors.

Submit your manuscript here: https://www.dovepress.com/risk-management-and-healthcare-policy-journal 